\title{
La prima salarial de las lenguas extranjeras en el mercado de trabajo español*
}

\author{
Victor Ginsburgh \\ ECARES, Université Libre de Bruxelles y \\ CORE, Université catholique de Louvain \\ Juan Prieto-Rodríguez \\ Universidad de Oviedo
}

\begin{abstract}
Resumen
En este trabajo se evalúa la prima salarial obtenida por los trabajadores españoles que utilizan alguna lengua extranjera en su puesto de trabajo. Para ello se ha definido una variable que mide la escasez relativa en la población española de los conocimientos linguiísticos utilizados por los trabajadores. El problema de la posible endogeneidad de los conocimientos de lenguas extranjeras se ha tratado explícitamente mediante un método iterativo de regresión cuantílica de variables instrumentales que es robusto ante la presencia de instrumentos débiles. Este procedimiento de estimación ha permitido analizar el efecto de las habilidades lingüísticas en distintos puntos de la distribución de salarios a partir de los datos del Panel de Hogares de la Unión Europea (ECHP).
\end{abstract}

Palabras clave: idiomas, primas salariales, regresiones cuantílicas

Clasificación JEL: J31, J24

\begin{abstract}
In this paper we evaluate the wage premium obtained by Spanish workers who use any foreign language in their job. To do so we have defined a variable that measures the relative lack among Spanish population of linguistic knowledge used by Spanish workers. The problem of the possible endogeneity of foreign languages knowledge has been treated explicitly by an iterative method of instrumental variables quantile regression that is robust to the presence of weak instruments. This estimation method allows us to analyze the effect of linguistic skills at different parts of the wage distribution using data from the European Union Household Panel (ECHP).
\end{abstract}

Keywords: languages, wage premium, quantile regressions

JEL Classification: J31, J24

\section{Introducción}

El desarrollo económico de España en los últimos cuarenta años ha estado vinculado de manera muy importante al sector exterior. Tanto el turismo, que sigue sien-

* Este trabajo se ha beneficiado de los fondos de los proyectos de investigación SEC2003-08397 y MEC-04-SEJ-04065. Asimismo, los autores quisieran agradecer los comentarios y sugerencias de Víctor Fernández. Con su ayuda el trabajo ha mejorado de manera importante. En cualquier caso, él no es responsable de los errores existentes, que son méritos exclusivos de los autores. 
do la principal industria nacional, como la creciente internacionalización de los mercados de las empresas españolas han requerido que algunos trabajadores nacionales utilizaran un segundo idioma en su trabajo y eliminar o mitigar, de esta forma, las barreras lingüísticas con los potenciales clientes (o proveedores). Así, la masiva implantación del inglés como segunda lengua en la enseñanza primaria y secundaria, y el descenso en la difusión del francés, pueden entenderse como una elección racional determinada por la mayor exposición al inglés, que se ha convertido en la lengua franca de la actualidad y es hablado y entendido por más de mil quinientos millones de personas en el mundo (Crystal, 2001).

En cualquier caso, los países en los que el inglés no es una de sus lenguas oficiales representan una gran parte de nuestros intercambios comerciales y, por tanto, los trabajadores españoles no sólo pueden mejorar su capital humano mediante el aprendizaje del inglés sino que también existen otros idiomas que pueden resultar necesarios en determinados puestos de trabajo. De hecho, la escasez relativa de personas que conozcan estas lenguas podría suponer unas mayores ganancias en el caso de producirse un emparejamiento adecuado en el mercado de trabajo ${ }^{1}$.

La literatura más importante sobre el impacto económico de los idiomas es la relativa al análisis del conocimiento y el uso por parte de los emigrantes de las lenguas oficiales de los países donde se establecen. Estos estudios se han centrado en países que tradicionalmente han recibido inmigrantes como Australia, Canadá, Alemania, Israel, el Reino Unido y los Estados Unidos ${ }^{2}$. Asimismo, también pueden encontrarse algunos trabajos que analizan el caso de los habitantes de sociedades multilingües como Canadá (Shapiro y Stelcner, 1997), Luxemburgo (Klein, 2003), Suiza (Cattaneo y Winkelman, 2003) y los Estados Unidos (Fry y Lowell, 2003).

Sin embargo, resulta sorprendente la escasez de trabajos relativos al impacto de las lenguas extranjeras sobre los salarios de los trabajadores nativos. Una excepción la constituye el trabajo de Williams (2005) sobre los países de la Unión Europea antes de su última ampliación (la Unión Europea a 15).

El objetivo de este trabajo es cubrir en parte esta laguna para el caso español y evaluar el rendimiento de los idiomas extranjeros en un país como España en el que existen amplias zonas donde las lenguas regionales conviven y compiten con el castellano como lenguas oficiales. Para ello se ha estimado, separadamente para hombres y mujeres, el efecto sobre los salarios del uso de lenguas extranjeras en el puesto de trabajo. Los datos utilizados para el análisis empírico son los del Panel de Hogares de la Unión Europea (ECHP) para el año 2001, que es el periodo más reciente para el que esta base de datos ofrece este tipo de información.

\footnotetext{
1 Por otro lado, podría esperarse que una mejora significativa de la difusión del castellano como lengua internacional redujera la ventaja económica de aprender otras lenguas en España.

2 Australia (Chiswick y Miller, 1995), Canada (Abbott y Beach, 1992, Aydemir y Skuterud, 2005, Chiswick y Miller, 1995), Alemania (Dustmann y Van Soest, 2002), Israel (Beenstock, Chiswick y Repetto, 2001, Berman, Lang y Siniver, 2003, Chiswick y Miller, 1995, Chiswick, 1998), Reino Unido (Leslie y Lindley, 2001), y los Estados Unidos (Bleakey y Chin, 2004, Bratsberg, Ragan y Nasir, 2002, Chiswick y Miller, 1995, 2002, Hellerstein y Neumark, 2003).
} 
En el análisis se ha intentado, en primer lugar, tener en cuenta la posible endogeneidad de las habilidades lingüísticas utilizadas por los trabajadores. Si la capacidad para aprender una segunda lengua está relacionada con la habilidad inobservada de los trabajadores, entonces es posible que la correlación entre las variables independientes de la ecuación salarial y el término de perturbación aleatoria no sea nula. Sin embargo, tal y como se argumenta más adelante, la variable lingüística utilizada en esta investigación no mide directamente el capital humano del trabajador, lo que limitaría este problema. En cualquier caso, se han estimado regresiones cuantílicas con variables instrumentales utilizando el método propuesto por Chernozhukov y Hansen (2004, 2005 y 2006), lo que permite estudiar cómo evoluciona el rendimiento de las lenguas extranjeras en el mercado de trabajo español en distintos puntos de la distribución de ingresos.

En segundo lugar, las lenguas extranjeras no se han incorporado separadamente mediante un conjunto de variables ficticias. Por contra, se ha preferido usar un indicador de la escasez relativa de los conocimientos lingüísticos de los trabajadores. Para cada lengua extranjera utilizada en el trabajo, se ha definido la razón de exclusión lingüística como el porcentaje de la población española que desconoce dicha lengua. Para los individuos que no usan idiomas extranjeros en su puesto de trabajo esta variable será igual a cero. Por el contrario, para aquéllos trabajadores que utilicen lenguas extranjeras esta variable tendrán un valor positivo que será mayor cuanto menor sea el número de hablantes de la misma en el conjunto de la población española. El parámetro asociado a esta variable permite recuperar el rendimiento de las lenguas extranjeras demandadas por las empresas cuando se conozca el porcentaje de la población que desconoce dicho idioma. $\mathrm{Si}$, como es de esperar, el parámetro estimado es positivo el rendimiento será mayor para aquellas lenguas que son menos comunes en España.

El trabajo se organiza como sigue. En la Sección 2, se discute el modelo a estimar y se revisan algunos problemas econométricos que tienen que ser afrontados en la estimación del mismo. En la Sección 3, se presenta la base de datos utilizada. En la Sección 4, se discuten los resultados obtenidos y, por último, la Sección 5 cierra la investigación con las principales conclusiones de la misma.

\section{El modelo empírico}

El objetivo de este trabajo es evaluar el efecto sobre los salarios del uso de lenguas extranjeras en el puesto de trabajo para los trabajadores españoles, tanto hombres como mujeres. En esta sección se presenta el modelo a estimar y se discuten algunos de los problemas econométricos del mismo. Dado que la mayor parte de la literatura sobre el rendimiento salarial de los idiomas se refiere a las habilidades lingüísticas de los emigrantes, este será el marco de referencia utilizado. La especificación usual en estos casos es una ecuación salarial de tipo minceriano que incluye variables lingüísticas y que se puede expresar como:

$$
\ln w_{i}=X_{i} \beta+L_{i} \gamma+u_{i}
$$


donde el subíndice $i$ representa al i-ésimo trabajador, el vector $\beta$ y el escalar $\gamma$ son los parámetros a estimar, $w_{i}$ es el salario por hora y $X_{i}$ el vector de variables explicativas, que se supone que se distribuyen independientemente del término de perturbación aleatoria, $u_{i}$. Para mantener las ecuaciones estimadas tan cerca como sea posible del modelo de Mincer, el vector $X_{i}$ incluye las siguientes variables de control: dos variables ficticias de nivel educativo que representan la enseñanza superior y la educación secundaria; los años de antigüedad en el puesto de trabajo y su cuadrado y los años de experiencia potencial y su cuadrado.

Una diferencia importante entre el modelo utilizado en esta investigación y otros más usuales se refiere a cómo se define la variable idiomática, $L_{i}$. Habitualmente esta variable está definida en términos de la habilidad lingüística declarada por el individuo, independientemente de que sea relevante o no en el puesto de trabajo. En los estudios sobre emigrantes puede suponerse que toda mejora en el uso de la lengua del país de residencia tendrá importancia en el mercado de trabajo: el grado de integración social y el éxito económico del emigrante aumentará a medida que se eleve su dominio de la lengua del país de residencia. Sin embargo, en esta investigación se trata de evaluar la prima salarial del uso de idiomas no oficiales para los trabajadores nacionales. En el ECHP, éstos declaran qué lenguas extranjeras utilizan en el puesto de trabajo. En este caso, declarar el uso de una determinada lengua supone tanto el conocimiento de la misma por parte del trabajador como la necesidad de usarla por parte de la empresa. Esto representa un requisito adicional por el lado de la demanda que elimina los problemas de sobrecualificación lingüística de los trabajadores y permite no subestimar la prima salarial para los trabajadores nacionales del uso de lenguas extranjeras en el puesto de trabajo ${ }^{3}$.

Asimismo, en la literatura sobre los conocimientos lingüísticos de los emigrantes, $L_{i}$ es, por lo general, una variable ficticia que toma el valor 1 si el nivel declarado de destreza en la lengua del país para el que se estima la ecuación salarial es igual o mayor que un determinado umbral y 0 en caso contrario. No obstante, en otros trabajos esta variable representa diferentes grados de habilidad declarada en la lengua oficial del país de destino ${ }^{4}$. Por otro lado, en los escasos trabajos que evalúan el rendimiento de las lenguas extranjeras para los trabajadores nacionales se suele incorporar una variable ficticia para cada idioma considerado (véase, por ejemplo, Galasi (2003) o Williams (2005)).

En esta investigación para tener en cuenta simultáneamente las distintas lenguas que podrían usarse en el puesto de trabajo no se ha incluido una variable ficticia por

3 La condición necesaria, aunque no suficiente, para observar valores de la variable $L_{i}$ distintos de cero es que la empresa demande habilidades lingüísticas a sus trabajadores. En este caso, y sólo en este caso, la empresa estará dispuesta a retribuir este tipo de habilidades. Sin embargo, si no se dispone de esta información y se utiliza como variable independiente en la ecuación (1) los conocimientos lingüísticos del trabajador, aunque no sean demandados por la empresa, se podría subestimarar el verdadero rendimiento de las habilidades lingüísticas en las empresas, al no distinguir los trabajadores que precisan hacer uso de las mismas de los que no lo hacen.

4 Véase, por ejemplo, Berman, Lang y Siniver (2003), Chiswick y Miller (2002) o Fry y Lowell (2003). 
idioma sino que se ha utilizado una medida de la escasez relativa de las lenguas que el individuo declara usar en su lugar de trabajo. Esta medida consiste en la razón de exclusión linguística (Ginsburgh y Weber, 2005), definida como el porcentaje de la población española que desconoce un determinado idioma, y está basada en los resultados de una encuesta encargada en el año 2000 por la Dirección de Educación y Cultura de la Unión Europea (Directorate of Education and Culture of the EU) ${ }^{5}$. Para cada uno de los países miembros en aquel momento de la Unión Europea se realizaron mil entrevistas sobre las habilidades lingüísticas de los individuos. La información usada por Ginsburgh y Weber se refiere a las respuestas relativas a las dos preguntas siguientes:

a) ¿Cuál es su lengua materna?

b) ¿Qué otras lenguas conoce usted?

Esta encuesta constituye una importante fuente de información sobre los conocimientos lingüísticos de los ciudadanos europeos y a partir de la respuesta a estas dos preguntas se puede calcular el grado de exclusión lingüístico de cada lengua para los países incluidos en la muestra ${ }^{6}$.

A partir de la información de esta encuesta, en esta investigación $L_{i}$ toma el valor 0 para los trabajadores que sólo usan las lenguas oficiales (incluso si trabajan fuera de la zona en la que estás son oficiales, por ejemplo un trabajador en Madrid que utilice el catalán o el gallego en su trabajo) ${ }^{7}$ y el valor de la ratio de exclusión lingüística correspondiente para los trabajadores que precisan alguno de los cuatro idiomas más extensamente hablados en la Unión Europea antes de su ampliación en 2004 (inglés, francés, alemán e italiano $)^{8}$. Las razones de exclusión lingüística para España relativas a estas cuatro lenguas se presentan en la Tabla 1.

TABLA 1

RATIOS DE EXCLUSIÓN LINGÜÍSTICA PARA ESPAÑA (PORCENTAJES)

\begin{tabular}{|c|c|c|c|}
\hline Inglés & Francés & Alemán & Italiano \\
\hline 64 & 81 & 98 & 98 \\
\hline
\end{tabular}

Fuente: Ginsburgh y Weber (2005, p. 279).

5 Véase INRA (2001).

6 Además esta base de datos fue realizada aproximadamente en el mismo momento en que se recogieron los datos del ECHP utilizados para las estimaciones de las ecuaciones salariales de este trabajo.

7 En la muestra española del ECHP, las preguntas relativas a las necesidades lingüísticas en el trabajo se refieren únicamente a las lenguas extranjeras, lo que impide realizar un análisis por Comunidades Autónomas y evaluar el rendimiento del bilingüismo en aquéllas que tienen dos lenguas oficiales.

8 Para aquellos trabajadores que declaran usar dos lenguas extranjeras, $L_{i}$ toma el valor de la razón de exclusión correspondiente al idioma más escaso. 
Como ya se ha señalado, dada esta definición de la variable $L_{i}$, el signo esperado de $\gamma$ es positivo: cuanto menos conocido sea un determinado idioma extranjero, es decir cuanto mayor sea el porcentaje de exclusión lingüística en España para un idioma, más alto es el rendimiento salarial esperado de dicha lengua. Esta forma de construir la variable $L_{i}$ tiene la ventaja de que todos los idiomas extranjeros se representan por una única variable, lo que permite abordar los problemas de endogeneidad de manera más sencilla. Además, el efecto de cada lengua puede ser recuperado fácilmente multiplicando la razón de exclusión lingüística de dicha lengua por el valor estimado de $\gamma$.

\section{Problemas econométricos}

En primer lugar, la estimación del rendimiento de las lenguas puede estar sujeto a un problema de endogeneidad similar al existente al evaluar el rendimiento de la educación: el conocimiento de idiomas y los ingresos pueden estar correlados con las habilidades y el talento inobservado de los trabajadores. Por tanto, la correlación entre las variables independientes, especialmente $L_{i}$, y el término de error de la ecuación (1) puede no ser nula. Pero, $\operatorname{si} \operatorname{corr}\left(L_{i}, u_{i}\right) \neq 0$ las estimaciones por mínimos cuadrados ordinarios serán inconsistentes. Además, es de esperar que, si esta correlación es positiva, las estimaciones mínimo cuadráticas estén sobreestimando los parámetros de interés.

La solución usualmente propuesta en la literatura es usar un estimador de variables instrumentales, lo que plantea la necesidad de establecer y valorar un buen instrumento para la variable lingüística de la ecuación (1). Chiswick y Miller (1995) han usado los siguientes instrumentos para la fluidez de los emigrantes en la lengua del país de destino: número y edad de los hijos, si el individuo se casó en el extranjero y la concentración de la lengua minoritaria en el lugar de residencia definida como la proporción de la población en la región de residencia del trabajador que declara conocer la misma lengua no oficial ${ }^{9}$. Dustmann y Van Soest (2002) usan el nivel de educación del padre. Por su parte, Bleakey y Chin (2004) usan como instrumento la edad del emigrante en el momento de llegar al país interaccionada con una variable ficticia que toma valor uno para los países de origen cuya lengua oficial no es el inglés, lo que permite tener en cuenta el posible efecto de una adaptación más fácil para aquellos emigrantes que se expatriaron a edades jóvenes. Galasi (2003) usa la nota obtenida en el examen de acceso a la enseñanza superior para analizar el rendimiento de los idiomas extranjeros en Hungría.

Sin embargo, en esta investigación la variable $L_{i}$ incorpora una exigencia del lado «de la demanda». Por tanto, aunque esté correlacionada con el término de error de la ecuación (1), se puede suponer que esta correlación no está ligada a la habilidad inobservada del trabajador ya que $L_{i}$ no es estrictamente una variable de capital humano. emigrante.

9 Chiswick y Miller también han usado como instrumento la lengua oficial del país de origen del 
Otro problema econométrico al que nos enfrentamos al estimar la ecuación (1) son los errores de medida relativos a las variables que suponen realizar una auto-evaluación por parte de los individuos. Estos errores de medida estarían relacionados con la heterogeneidad inobservada de los trabajadores a la hora de evaluar sus conocimientos lingüísticos y pueden considerarse persistentes en el tiempo. En nuestro caso, dado que $L_{i}$ representa la escasez relativa de la lengua extranjera usada en el puesto de trabajo, y no las capacidades lingüísticas declaradas de manera subjetiva por los trabajadores, hemos supuesto que los errores de medida inherentes a esta variable no son persistentes en el tiempo ya que la respuesta a la pregunta planteada en el ECHP puede considerarse objetiva.

Por otro lado, $L_{i}$ puede variar de un año a otro fundamentalmente por tres motivos: (a) cambios dentro de la empresa que modifican las necesidades lingüísticas a ser cubiertas por los trabajadores (por ejemplo, la empresa comienza a exportar a nuevos mercados o deja de hacerlo); (b) cambios de empresa de los trabajadores que supongan cambios en las exigencias lingüísticas y (c) errores de medida no persistentes en el tiempo. La Tabla 2 recoge los cambios en esta variable entre las dos últimas olas del ECHP.

TABLA 2

TABLA DE TRANSICIÓN DE LA VARIABLE $L$ ENTRE 2000 Y 2001 (PORCENTAJES)

\begin{tabular}{|l|c|c|c|c|}
\hline \multirow{2}{*}{} & \multicolumn{3}{|c|}{$\boldsymbol{L}_{i, t-\mathbf{l}}$} \\
\cline { 2 - 5 } $\boldsymbol{L}_{\boldsymbol{i}, t}$ & $\begin{array}{c}\mathbf{0} \\
\text { (Español) }\end{array}$ & $\begin{array}{c}\mathbf{0 , 6 4} \\
\text { (Inglés) }\end{array}$ & $\begin{array}{c}\mathbf{0 , 8} \\
\text { (Francés) }\end{array}$ & $\begin{array}{c}\text { 0,98 } \\
\text { (Italiano } \\
\text { o Alemán) }\end{array}$ \\
\hline Hombres & & & & \\
0 (Español) & 91,88 & 5,12 & 2,22 & 0,78 \\
0,64 (Inglés) & 40,00 & 51,58 & 6,32 & 2,11 \\
0,81 (Francés) & 30,30 & 12,12 & 51,52 & 6,06 \\
0,98 (Italiano o Alemán) & 45,45 & 9,09 & 18,18 & 27,27 \\
\hline Total & 88,02 & 7,54 & 3,36 & 1,08 \\
\hline Mujeres & & & & 0,42 \\
0 (Español) & 90,70 & 5,64 & 3,24 & 1,37 \\
0,64 (Inglés) & 34,25 & 57,53 & 6,85 & 6,52 \\
0,81 (Francés) & 30,43 & 13,04 & 50,00 & 50,00 \\
0,98 (Italiano o Alemán) & 40,00 & 10,00 & 0,00 & 1,20 \\
\hline Total & 83,89 & 9,48 & 5,43 & \\
\hline
\end{tabular}


Para tratar estos errores de medida no persistentes en el tiempo hemos seguido el procedimiento utilizado por Dustmann y Van Soest (2002) y hemos utilizado como instrumento la variable $L_{i t}$ rezagada un periodo.

Lamentablemente, el Panel de Hogares de la Unión Europea no contiene ninguna variable que pueda ser usada como instrumento para corregir la heterogeneidad inobservada invariante en el tiempo ${ }^{10}$. Sin embargo, los resultados de Dustmann y Van Soest (2002) indican que corregir por los errores de medida no sistemáticos (utilizando como instrumentos rezagos de la variable endógena) produce resultados que no difieren significativamente de los obtenidos cuando se trata de corregir los errores sistemáticos (utilizando la educación de los padres como instrumento). Además, como se indicó anteriormente, es de esperar que este tipo de endogeneidad no sea un problema importante en esta investigación dado cómo se ha definido la variable lingüística, que implica la existencia de requisitos sobre qué idiomas extranjeros se deben usar en la empresa.

\section{Los datos: el Panel de Hogares de la Unión Europea (ECHP)}

Como ya se ha señalado, para el análisis empírico se han utilizado los micro-datos de la última ola del Panel de Hogares de la Unión Europea (ECHP), llevada acabo para España por el INE en colaboración con la Oficina Estadística del la Unión Europea (EUROSTAT). Esta encuesta incluye datos relativos a la situación socio-económica de los individuos mayores de 16 años agrupados por hogares, de los que se conocen sus características personales, familiares y laborales. Asimismo, se conoce la estructura familiar completa, incluyendo las características de los miembros menores de 16 años.

Entre 1994 y 1999, las preguntas relativas a los requisitos lingüísticos del puesto de trabajo se referían a la necesidad de utilizar lenguas distintas de las oficiales en la ocupación principal y se identificaban hasta tres idiomas no oficiales utilizados por el trabajador. Sin embargo, en los años 2000 y 2001 se modificó el cuestionario y estas preguntas se formularon de manera diferente y fueron trasladadas a la parte del cuestionario relativa a la educación. La pregunta pasó a referirse, para la submuestra española, a las lenguas extranjeras principal y secundaria usadas en el trabajo principal.

Dada la heterogeneidad introducida por los cambios en el cuestionario, se ha considerado inadecuado combinar ambas partes de la base de datos y se optó por utilizar únicamente la información de las dos últimas olas del ECHP.

Asimismo, se ha excluido de la muestra a los inmigrantes, ya que los mecanismos que gobiernan la adquisición de lo que para los trabajadores españoles son lenguas ex-

10 Se trató de estimar el modelo para la submuestra de trabajadores casados o que conviven con una pareja estable, utilizando el nivel de educación y/o los requisitos lingüísticos en el trabajo de los cónyuges como instrumento. Sin embargo, los resultados obtenidos no fueron satisfactorios. 
tranjeras puede ser completamente distintos para los trabajadores no nativos. El trabajo se centra, por tanto, en el rendimiento de las lenguas extranjeras para los trabajadores nacionales en el mercado de trabajo español.

Tal y como muestra la Tabla 3, en España el 85\% de los trabajadores que declaran usar algún idioma extranjero en su trabajo utilizan el inglés. Esta lengua constituye, por tanto, el idioma extranjero dominante en el mercado de trabajo español seguido, a mucha distancia, por el francés. El resto de idiomas tienen una presencia poco más que testimonial.

TABLA 3

LENGUAS EXTRANJERAS UTILIZADAS POR LOS TRABAJADORES NATIVOS EN ESPAÑA (POR SEXO)

\begin{tabular}{|c|c|c|c|c|c|c|c|c|}
\hline \multirow[b]{2}{*}{$\begin{array}{l}\text { Lengua extranjera } \\
\text { principal usada } \\
\text { en el trabajo }\end{array}$} & \multicolumn{8}{|c|}{ Segunda lengua extranjera usada en el trabajo } \\
\hline & $\begin{array}{c}\text { Sólo una } \\
\text { lengua }\end{array}$ & Inglés & Francés & Alemán & Italiano & Portugués & Ruso & Total \\
\hline $\begin{array}{l}\text { Hombres }(\mathrm{N}=2372) \\
\text { Inglés } \\
\text { Francés } \\
\text { Alemán } \\
\text { Italiano }\end{array}$ & $\begin{array}{r}181 \\
34 \\
6 \\
2\end{array}$ & $\begin{array}{l}6 \\
1 \\
2\end{array}$ & 45 & $\begin{array}{l}9 \\
1\end{array}$ & $\begin{array}{l}3 \\
1\end{array}$ & 2 & 1 & $\begin{array}{r}241 \\
42 \\
7 \\
4\end{array}$ \\
\hline Total & 223 & 9 & 45 & 10 & 4 & 2 & 1 & 294 \\
\hline $\begin{array}{l}\text { Mujeres (N=1448) } \\
\text { Inglés } \\
\text { Francés } \\
\text { Alemán }\end{array}$ & $\begin{array}{r}133 \\
30 \\
1\end{array}$ & $\begin{array}{l}9 \\
2\end{array}$ & 30 & $\begin{array}{r}13 \\
1\end{array}$ & $\begin{array}{l}3 \\
3\end{array}$ & & 1 & $\begin{array}{r}180 \\
43 \\
3\end{array}$ \\
\hline Total & 161 & 11 & 30 & 14 & 6 & 0 & 1 & 226 \\
\hline
\end{tabular}

Se aprecian, igualmente, diferencias entre hombres y mujeres. Así, la proporción de hombres que usan lenguas extranjeras en su puesto de trabajo (12,5 por ciento) es menor que la de mujeres (15,7 por ciento). Más aún, las mujeres que utilizan al menos dos idiomas extranjeros representan un mayor porcentaje que los hombres (29 por ciento frente al 24).

La Tabla 4 permite comparar la situación en España con los países de la Unión Europea incluidos en el ECHP para los que se dispone de información sobre las lenguas habladas en el puesto de trabajo, excluyendo Bélgica por su especial situación de bilingüismo.

El primer dato a resaltar es que la media de la variable $L_{i}$, tanto para los hombres como las mujeres, toma valores muy bajos para España. En cualquier caso, estas ci- 


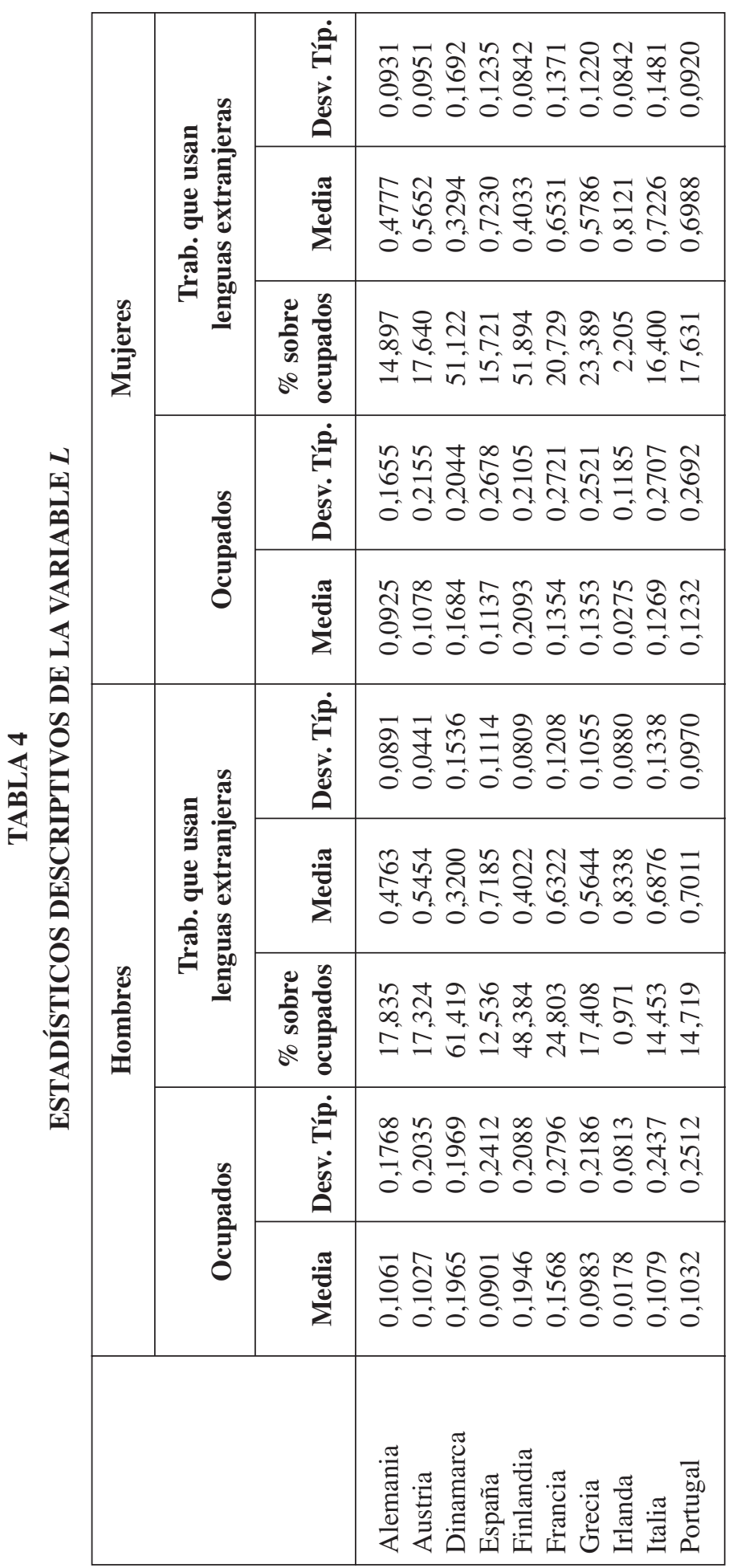


fras, aunque se encuentran en la franja baja, no difieren mucho de los observados para otros países mediterráneos, Alemania o Austria.

Sin embargo, un análisis más detallado nos lleva a un diagnóstico preocupante. La media de esta variable depende de dos factores: el número de trabajadores que hablan lenguas extranjeras en su puesto de trabajo y la escasez relativa de los conocimientos de idiomas en cada país. En el caso español, la media observada de $L_{i}$ obedece a la exigua proporción de trabajadores que declaran utilizar alguna lengua extranjera en su puesto de trabajo combinado con una escasez relativa de conocimientos de idiomas, lo que lleva a que la ratio de exclusión lingüística para los más importantes idiomas modernos tome valores superiores a los observados en otros países (véase Ginsburgh y Weber, 2005). Por contra, en otros países, salvo Irlanda, el uso de idiomas no oficiales en el puesto de trabajo es más común que en España y los conocimientos de idiomas de sus poblaciones son mayores, tal y como indica la media condicionada de $L_{i}$ a usar lenguas extranjeras en el trabajo. Los países mediterráneos son los que presentan una situación más parecida a la española, mientras que los dos países nórdicos (Dinamarca y Finlandia) representan casos totalmente distintos con altas tasas de uso de idiomas extranjeros por parte de los trabajadores y una mayor difusión de los conocimientos lingüísticos.

Respecto a las diferencias entre hombres y mujeres, estas son favorables a las mujeres en el caso español, tal y como puede verse en la Tabla 5.

TABLA 5

PRUEBA T DE IGUALDAD DE MEDIAS ENTRE HOMBRES Y MUJERES PARA LA VARIABLE $L$

\begin{tabular}{|c|c|c|c|c|c|c|c|}
\hline & \multirow[b]{2}{*}{$\begin{array}{l}\text { Diferencia } \\
\text { de medias }\end{array}$} & \multirow[b]{2}{*}{$\begin{array}{l}\text { Desviación } \\
\text { típica } \\
\text { diferencia } \\
\text { de medias }\end{array}$} & \multicolumn{2}{|c|}{$\begin{array}{c}\text { Intervalo de } \\
\text { confianza }(95 \%)\end{array}$} & \multicolumn{3}{|c|}{$\operatorname{Pr}(\mathrm{T}<\mathrm{t})$} \\
\hline & & & $\begin{array}{c}\text { Límite } \\
\text { inferior } \\
\text { superior }\end{array}$ & Límite & $\begin{array}{l}\text { Ho:dif }=0 \\
\text { Ha:dif }<0\end{array}$ & $\begin{array}{l}\text { Ho:dif }=0 \\
\text { Ha:dif } \neq 0\end{array}$ & $\begin{array}{l}\text { Ho:dif }=0 \\
\text { Ha:dif }>0\end{array}$ \\
\hline Alemania & 0,013639 & 0,005252 & 0,003343 & 0,023934 & 0,9953 & 0,0094 & 0,0047 \\
\hline Austria & $-0,005083$ & 0,009475 & $-0,023667$ & 0,013501 & 0,2959 & 0,5917 & 0,7041 \\
\hline Dinamarca & 0,028113 & 0,008961 & 0,010539 & 0,045686 & 0,9991 & 0,0017 & 0,0009 \\
\hline España & $-0,023589$ & 0,008558 & $-0,040369$ & $-0,006808$ & 0,0029 & 0,0059 & 0,9971 \\
\hline Finlandia & $-0,014686$ & 0,009893 & $-0,034089$ & 0,004716 & 0,0689 & 0,1378 & 0,9311 \\
\hline Francia & 0,021417 & 0,010016 & 0,001777 & 0,041056 & 0,9837 & 0,0326 & 0,0163 \\
\hline Grecia & $-0,037075$ & 0,010604 & $-0,057875$ & $-0,016275$ & 0,0002 & 0,0005 & 0,9998 \\
\hline Irlanda & $-0,009733$ & 0,005490 & $-0,020505$ & 0,001039 & 0,0383 & 0,0765 & 0,9610 \\
\hline Italia & $-0,018942$ & 0,008698 & $-0,035997$ & $-0,001887$ & 0,0148 & 0,0295 & 0,9852 \\
\hline Portugal & $-0,020016$ & 0,008273 & $-0,036236$ & $-0,003796$ & 0,0078 & 0,0156 & 0,9922 \\
\hline
\end{tabular}


Además, esta diferencia es significativamente distinta de cero. Hay que señalar que esta situación es semejante a la existente en el resto de países mediterráneos: Grecia, Italia y Portugal. Por su parte, las diferencias observadas favorables a las mujeres no resultaron significativas para Austria, Finlandia e Irlanda. Por último, la diferencia de medias de $L_{i}$ resultó favorables a los hombres y estadísticamente significativas para Alemania, Dinamarca y Francia. En cualquier caso, el perfil común de los países mediterráneos parece indicar una mayor especialización lingüística en el mercado de trabajo de las mujeres en estos países. Dados los mayores índices de segregación ocupacional de las mujeres en estos países y su escaso acceso a las ocupaciones más elevadas, se puede suponer que en estos países se ha optado por el uso de personal administrativo especializado que limite los problemas lingüísticos de los directivos.

\section{Resultados}

Tradicionalmente las evaluaciones del rendimiento salarial de los idiomas se han basado en estimaciones de ecuaciones salariales por mínimos cuadrados ordinarios o, en caso de considerar explícitamente el problema de endogeneidad, han utilizado algún estimador de variables instrumentales. Sin embargo, en este trabajo se ha optado por estimar las ecuaciones salariales mediante regresión cuantílica. Esta técnica permite conocer cómo cambia el rendimiento de los idiomas extranjeros en los distintos puntos de la distribución condicionada del logaritmo natural de los salarios ${ }^{11}$.

Además, dado que se puede suponer que los trabajadores en el mismo cuantíl de la distribución condicionada del salario tienen características inobservadas similares, que pueden ser distintas a las de otros cuantiles, la regresión cuantílica puede ayudar a controlar la heterogeneidad inobservada y limitar el sesgo de la habilidad. Así, un aumento del rendimiento estimado de las lenguas extranjeras a lo largo de la distribución salarial podría interpretarse como evidencia a favor de una correlación positiva entre las habilidades lingüísticas y la habilidad inobservada.

En cualquier caso, aunque la regresión cuantílica puede limitar el problema de la endogeneidad de la variable lingüística, especialmente cuando ésta únicamente recoge capital humano, no está claro que lo elimine totalmente. En consecuencia, se ha optado por utilizar el estimador de regresión cuantílica con variables instrumentales propuesto por Chernozhukov y Hansen $(2004,2005,2006)$. Este estimador se caracteriza por poder ser implementado mediante un proceso iterativo y por ser robusto bajo identificación débil.

Este estimador se ha aplicado, separadamente, a las muestras de hombres y mujeres utilizando la variable $L_{i}$ rezagada un periodo como instrumento. La Figura 1 presenta de forma gráfica los resultados para diecinueve cuantiles $(0.05,0.10 \ldots, 0.90$,

11 Véase Buchinsky (1998) y Koenker y Hallock (2001) para una introducción a la regresión cuantílica. 
0.95). La línea continua representa la prima salarial estimada para la razón de exclusión lingüística de las lenguas utilizadas en el mercado de trabajo. Las regiones sombreadas representan los intervalos de confianza al 95 por ciento alrededor de las estimaciones de regresión cuantílica. Para facilitar la comparación con los métodos usuales de estimación en la media, las líneas horizontales discontinuas representan el coeficiente estimado por el método generalizado de los momentos y las líneas punteadas delimitan su intervalo de confianza al 95 por ciento.

\section{FIGURA 1 \\ REGRESIÓN CUANTÍLICA}

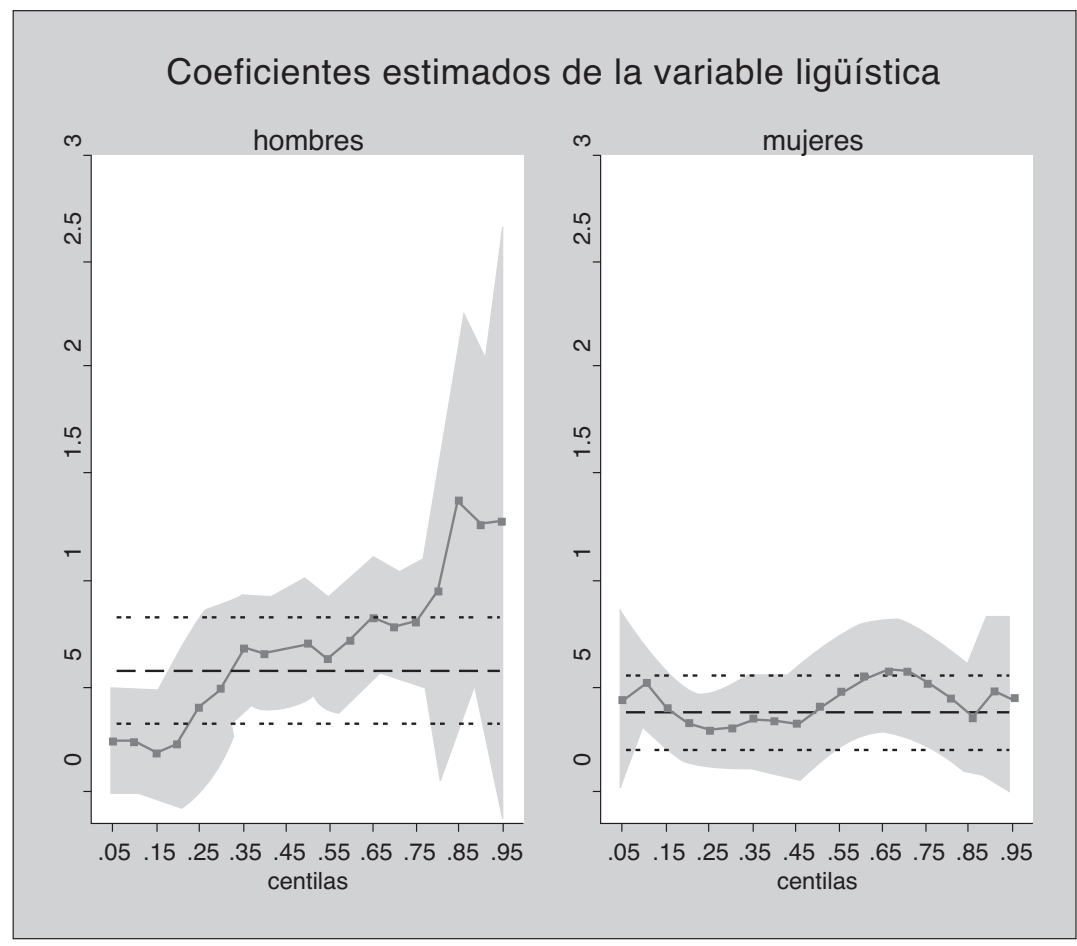

Nota: Las regiones sombreadas representan los intervalos de confianza del 95 por ciento alrededor de las estimaciones de regresión cuantílica con variables instrumentales. Las líneas horizontales discontinuas representan el coeficiente estimado por variables instrumentales y las líneas punteadas delimitan su intervalo de confianza al 95 por ciento.

Los coeficientes estimados de las regresiones cuantílicas tienen una interpretación similar a la de los coeficientes estimados por mínimos cuadrados ordinarios: miden el efecto marginal sobre el cuantíl correspondiente de un cambio unitario de la variable independiente. En nuestro caso, representan, en los distintos cuantiles, el incremento porcentual esperado de los salarios para aquellos trabajadores que pasen a utilizar en su puesto de trabajo una lengua no oficial cuya razón de exclusión lingüística 
sea uno. Tanto para los hombres como las mujeres, los efectos estimados son siginificativamente distintos de cero para todos los cuantiles excepto los más bajos de la distribución salarial de los trabajadores varones. Este resultado, por tanto, muestra que el uso de idiomas extranjeros en el trabajo incrementa el salario de manera significativa.

Para las mujeres, la prima salarial es estable a lo largo de toda la distribución de salarios, lo que implica que el rendimiento porcentual es similar para todos los cuantiles. Además, las estimaciones de regresión cuantílica se encuentran dentro del intervalo de confianza al 95 por ciento de la estimación por el método generalizado de los momentos (líneas punteadas); salvo alrededor del centil 70. En consecuencia, la heterogeneidad a lo largo de la distribución de la prima salarial de las lenguas extranjeras obtenida por las mujeres es tan reducida que la estimación en la media por GMM representa de manera apropiada lo que ocurre a lo largo de toda la distribución.

Por el contrario, se observa un rendimiento creciente del uso de idiomas extranjeros para los trabajadores varones a lo largo de la distribución de salarios. Asimismo, se produce un aumento en la varianza de las estimaciones a medida que nos movemos hacia los cuantiles superiores, lo que indica un serio problema de heterocedasticidad para las estimaciones mínimo cuadráticas. En conclusión, las estimaciones por el método generalizado de los momentos no son capaces de capturar la heterogeneidad en el rendimiento para los trabajadores varones del uso de idiomas: tanto para los cuantiles más bajos como más altos de la distribución de salarios el coeficiente estimado de regresión cuantílica está fuera del intervalo de confianza del estimador GMM.

Si se comparan los resultados obtenidos para hombres y mujeres se observa que el rendimiento porcentual estimado para los hombres es superior al de las mujeres a partir del centil 20. Del mismo modo, destaca el fuerte crecimiento de esta diferencia en el cuarto superior de la distribución salarial. Los hombres situados en el cuartil superior de la distribución salarial y que desempeñan, por tanto, las mejores ocupaciones, tienen una prima salarial que les permite prácticamente duplicar sus ingresos cuando utilizan el inglés en su puesto de trabajo. Sin embargo, para las mujeres situadas en el tramo alto de la distribución salarial este incremento supone una subida de poco más del treinta por ciento ${ }^{12}$. En consecuencia, parece que la mejora salarial (y profesional) asociada al uso de idiomas extranjeros en el puesto de trabajo es mucho menor en el caso de las mujeres, especialmente en los niveles más altos de la escala salarial respectiva. Por tanto, estos resultados aportan nueva evidencia empírica, aunque parcial por referirse al rendimiento de las lenguas extranjeras, a favor de la hipótesis del techo de cristal, ya que las mujeres parecen tener dificultades para rentabilizar de igual manera que los varones sus conocimientos lingüísticos, especialmente cuanto se encuentran en los cuantiles superiores de la distribución salarial.

Para poner estos resultados sobre la prima salarial porcentual en relación con los

12 Para obtener la prima salarial de cada idioma debe multiplicarse el coeficiente estimado por la ratio de exclusión lingüística respectiva. 
salarios percibidos, en la Figura 2 se ha representado la distribución salarial condicionada en el sexo y el uso de lenguas extranjeras para los trabajadores por cuenta ajena en el año 2001. Puede observarse que los varones que utilizan idiomas extranjeros en su trabajo constituyen el grupo con salarios más altos a lo largo de toda la distribución. Por el contrario, las mujeres que ocupan puestos de trabajo donde no se requieren conocimientos lingüísticos son el grupo peor pagado.

FIGURA 2

SALARIO POR SEXO Y USO DE LENGUAS EXTRANJERAS EN EL TRABAJO

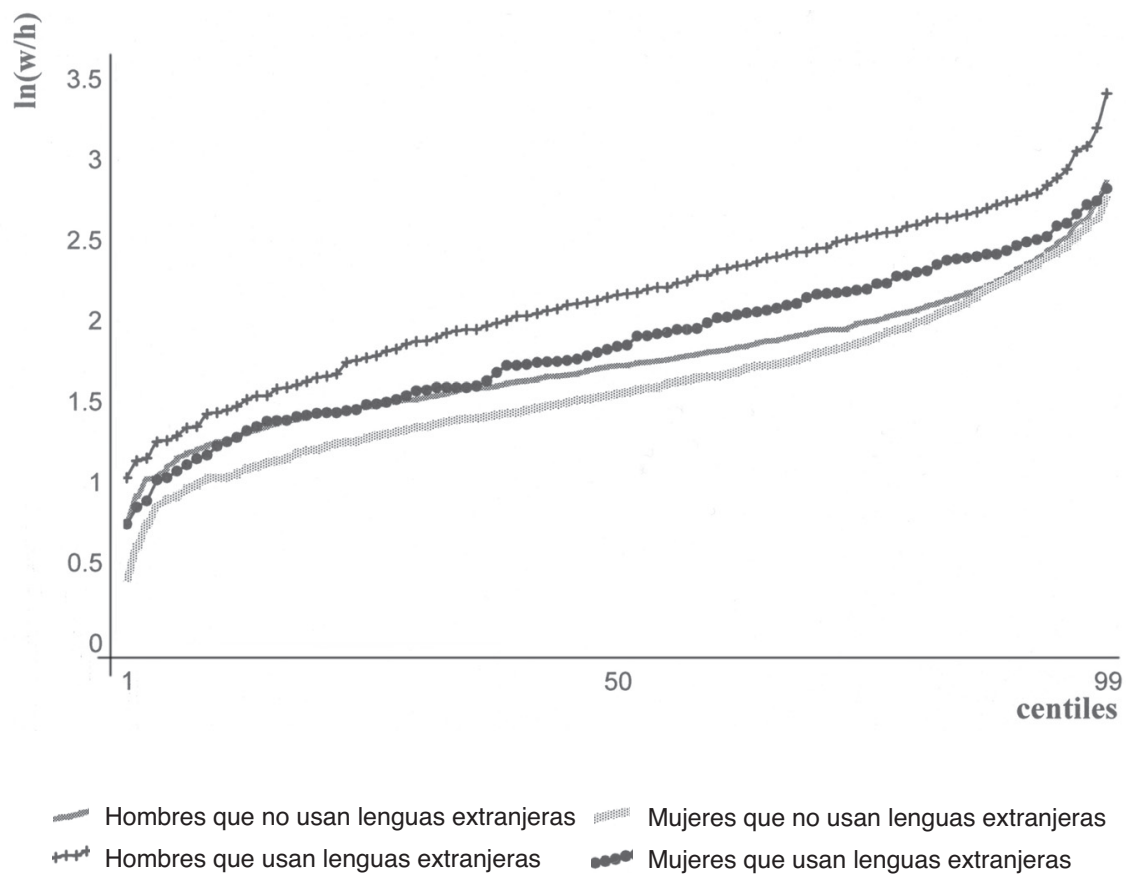

Además, para los hombres la ventaja de hablar alguna lengua extranjera en el trabajo aumenta a lo largo de la distribución salarial (con un pequeño bache alrededor del tercer cuartil). Sin embargo, para las mujeres esta diferencia crece en la parte baja de la distribución salarial, alcanzando el máximo alrededor del centil 70, y disminuye a medida que nos movemos hacia la parte más alta de la distribución salarial. Aquellas mujeres con mayores salarios, y mayor éxito profesional, acceden a ocupaciones para las que los conocimientos linguísticos no son un requisito imprescindible, ya que los salarios más altos de aquéllas que no utilizan sus conocimientos lingüísticos en el trabajo son comparables con los sueldos de las trabajadoras por cuenta ajena que sí los utilizan. Más aún, en la parte alta de la distribución, éstos no difieren de los salarios de los trabajadores varones que no hacen uso de lenguas extranjeras en sus 
puestos de trabajo. Sin embargo, sí hay una importante brecha entre los salarios de las mujeres y hombres que utilizan idiomas extranjeros, confirmando la idea de la existencia de un «techo de cristal» que limita el acceso a las mujeres a las ocupaciones más altas incluso para aquellas trabajadoras para las que el conocimiento de otras lenguas no supone una limitación.

\section{Conclusiones}

En lugar de incluir en las ecuaciones estimadas una variable ficticia para cada lengua, en esta investigación se utiliza como variable independiente una medida de la escasez relativa de las lenguas usadas en el mercado de trabajo (la ratio exclusión lingüística). Esta forma de definir la variable lingüística implica que el rendimiento disminuye a medida que la ratio de exclusión lingüística disminuye. Así, dado que el inglés es la lengua más conocida, su rendimiento será menor que el potencialmente obtenido por aquellos trabajadores que utilicen en su trabajo otras lenguas menos conocidas.

Esta variable lingüística permite reducir los problemas de endogeneidad asociados a la habilidad inobservable del trabajador y los errores de medida persistentes en el tiempo. Sin embargo, los errores de medida no sistemáticos pueden llevar a que las estimaciones por mínimos cuadrados ordinarios subestimen de manera significativa el parámetro de interés ${ }^{13}$. Por ello, se ha querido corregir este problema utilizando un estimador de variables instrumentales. Asimismo, se ha preferido estimar regresiones cuantílicas para analizar los cambios de la prima salarial de los idiomas en el mercado de trabajo español a lo largo de toda la distribución salarial y no únicamente en la media de la misma. Estos dos requisitos nos han llevado a seleccionar el estimador de regresión cuantílica con variables instrumentales propuesto por Chernozhukov y Hansen $(2004,2005$, 2006).

Los resultados obtenidos muestran que el mercado de trabajo retribuye positiva y significativamente el uso de lenguas extranjeras en el puesto de trabajo, tanto a los hombres como a las mujeres. Además, los rendimientos estimados, que se refieren a trabajadores nativos, son superiores a los rendimientos estimados en la mayor parte de los estudios sobre la habilidad y el uso de la lengua oficial del país de destino de los emigrantes.

Asimismo, se han detectado importantes diferencias por sexo. En primer lugar, las mujeres utilizan en mayor medida idiomas no oficiales en sus trabajos. Además, se ha observado que la diferencia en la media de la variable lingüística para hombres y mujeres es estadísticamente significativa, lo que parece indicar una mayor especialización lingüística en el mercado de trabajo de las mujeres. En segundo lugar, la estimación por cuantiles sugiere que el rendimiento es mayor en la parte alta de la distribución salarial de los hombres, mientras que se mantiene estable a lo largo de toda

13 Véase, por ejemplo, Dustmann y Van Soest (2001) o Bleakey y Chin (2004). 
la distribución para las mujeres. Además, para la mayor parte de los cuantiles, especialmente a medida que nos movemos hacia la parte alta de la distribución de salarios, los rendimientos del uso de idiomas (y las mejoras profesionales asociadas) son mayores para los hombres.

En consecuencia, estas diferencias en las retribuciones, unidas a los mayores índices de segregación ocupacional de las mujeres y a su mayor especialización en el uso de idiomas en el mercado laboral, nos levan a suponer que en España se ha optado por el uso de personal administrativo femenino que atenúa los problemas lingüísticos de algunos directivos. Esto supone nueva evidencia en favor de la existencia de un techo de cristal en el mercado de trabajo español, que impide que las mujeres alcancen los mismos niveles ocupacionales que los hombres.

\section{Referencias bibliográficas}

[1] ABBOTT, M. y C. BEACH (1992): «Immigrant earnings differentials in Canada: A more general specification of age and experience effects», Empirical Economics, 17, 221-238.

[2] ANGRIST, J. y A. KRUEGER (1991): «Does compulsory school attendance affect schooling and earnings?», Quarterly Journal of Economics 106, 979-1014.

[3] ASHENFELTER, O. y A. KRUEGER (1994): «Estimates of the economic return to schooling from a new sample of twins», American Economic Review, 84, 1157-1173.

[4] AYDEMIR, A. y M. SKUTERUD (2005): «Explaining the deterioration entry earnings of Canada's immigrant cohorts», 1996-2000, Canadian Journal of Economics, 38, 641-672.

[5] BEENSTOCK, M., B. CHISWICK y G. REPETTO (2001): «The effect of language distance and country of origin on immigrant language skills: Application to Israel, International Migration», 39, 33-62.

[6] BERMAN, E., K. LANG y E. SINIVER (2003): «Language skill complementarity: Returns to immigrant language acquisition», Labour Economics, 10, 265-290.

[7] BLEAKEY, H. y A. CHIN (2004): «Language skills and earnings: Evidence from childhood immigrants», The Review of Economics and Statistics, 86, 481-496.

[8] BRATSBERG, B., J. RAGAN y Z. NASIR (2002): «The effect of naturalization on wage growth: A panel study of young male immigrants», Journal of Labor Economics, 20,568-597.

[9] BUCHINSKY, M. (1998): «Recent advances in quantile regression models: A practical guideline for empirical research», The Journal of Human Resources, 33, 88-126.

[10] CATTANEO, A. y R. WINKELMANN (2003): «Earning differentials between German and French speakers in Switzerland», Working Paper 0309, University of Zürich.

[11] CHERNOZHUKOV, V. y C. HANSEN (2004): «The effects of 401(k) participation on wealth distribution: An instrumental quantile regression analysis», the Review of Economics and Statistics, 86(3), 735-751.

[12] CHERNOZHUKOV, V. y C. HANSEN (2005): «An IV model of quantile treatment effects», Econometrica, 73, 245-261. 
[13] CHERNOZHUKOV, V. y C. HANSEN (2006): «Instrumental quantile regression inference for structural and treatment effect models», Journal of Econometrics, 73, 245261.

[14] CHISWICK, B. (1998): «Hebrew language usage: Determinants and effects on earnings among immigrants in Israel», Journal of Population Economics, 15, 253-271.

[15] CHISWICK, B., Y. LEE y P. MILLER (2003): «Immigrants language skills: the Australian experience in a longitudinal survey», Annales d'Economie et de Statistique, 71-72, 97-129.

[16] CHISWICH, B. y P. MILLER (1995): «The endogeneity between language and earnings: international analyses», Journal of Labor Economics, 11, 246-288.

[17] CHISWICH, B. y P. MILLER (1996): «Ethnic networks and language proficiency among immigrants», Journal of Population Economics, 9, 19-35.

[18] CHISWICH, B. y P. MILLER (2002): «Immigrant learning: Language skills, linguistic concentrations and the business cycle», Journal of Population Economics, 15, 3157.

[19] CRYSTAL, D. (2001): A Dictionary of Language, Chicago: Chicago University Press.

[20] DUSTMANN, C. (1994): «Speaking fluency, writing fluency and earnings of migrants», Journal of Population Economics, 7, 133-156.

[21] DUSTMANN, C. y A. VAN SOEST (2001): «Language fluency and earnings: Estimators with miscalssified language indicators», Review of Economics and Statistics, 83, 663-674.

[22] DUSTMANN, C. y A. VAN SOEST (2002): «Language and the earnings of immigrants», Industrial and Labor Relations Review, 55, 473-492.

[23] FRY, RICHARD y B. LINDSAY LOWELL (2003): «The value of bilingualism in the U.S. labor market», Industrial and Labor Relations Review, 57, 128-140.

[24] GALASI, P. (2003): «Estimating wage equations for Hungarian higher education graduates», manuscript.

[25] GINSBURGH, V. y S. WEBER (2005): «Language Disenfranchisement in the European Union», Journal of Common Market Studies, 43, 273-286.

[26] HELLERSTEIN, J. y NEUMARK, D. (2003): «Ethnicity, language, and workplace segregation: Evidence from a new matched employer-employee data set», Annales d'Economie et de Statistique, 71/72, 19-78.

[27] INRA (2001): «Eurobaromètre 54 Special, Les Européens et les langues» , Février.

[28] KLEIN, C. (2003): «La valorisation des compétences linguistiques sur le marché du travail luxembourgeois», CEPS/INSTEAD Paper, Luxemburg.

[29] KOENKER, R. y K. HALLOCK (2001): «Quantile regression», Journal of Economic Perspectives, 15, 143-156.

[30] LESLIE, D. y J. LINDLEY (2001): «The impact of language ability on employment and earnings of Britain's ethnic communities», Economica, 68, 587-606.

[31] SHAPIRO, D. y STELCNER, M. (1997): «Language earnings in Quebec: Trends over twenty years, 1970-1990», Canadian Public Policy, 23, 115-140.

[32] WILLIAMS, D. (2005): «The economic returns to multiple language usage in Europe», CEPS/INSTEAD Paper, Luxemburg. 